\title{
Experimentos da Proposta de Integração SOFTWAY4IoT e FIWARE-Lab@RNP para Internet das Coisas (IoT)
}

\author{
Antonio Oliveira-Jr ${ }^{1,2}$, Ciro Macedo ${ }^{1}$, Gabriel M. F. de Almeida ${ }^{1}$, Leonardo Rodrigues ${ }^{1}$, \\ Marcos Abreu $^{1}$, Sand Correa ${ }^{1}$, Kleber Cardoso ${ }^{1}$ \\ ${ }^{1}$ Instituto de Informática (INF) - Universidade Federal de Goiás (UFG) \\ Goiânia - GO - Brasil \\ ${ }^{2}$ Fraunhofer Portugal AICOS \\ Porto - Portugal \\ \{antoniojr,kleber, sandluz\}@ufg.br, ciro.macedodifg.edu.br, \\ \{gabrielmatheus, marcosabreu, leonardorodriguesfurtado\}@inf.ufg.br
}

\begin{abstract}
It is expected that the number of Internet of Things objects will grow exponentially, consequently increasing the data flow traveling through the network. To support this increase, the diversity of technologies and the possible geographic distribution of these connected objects, an infrastructure capable of managing these demands is necessary. This work presents the details of the implementation and the integration experiments of SOFTWAY4IoT carried out together with the computational infrastructure (virtual machines) provided by RNP and the FIWARE-Lab@RNP platform. The use case is smart bins for collecting data about the amount of organic waste and monitoring garbage levels in selective waste bins.
\end{abstract}

Resumo. Espera-se que a quantidade de objetos da Internet das Coisas cresça de forma exponencial, aumentando consequentemente o fluxo de dados trafegados pela rede. Para suportar este aumento, a diversidade de tecnologias e a possível distribuição geográfica destes objetos conectados, é necessário uma infraestrutura apta a gerenciar estas demandas. Este trabalho apresenta os detalhes da implantação e os experimentos de integração do SOFTWAY4IoT realizado junto à infraestrutura computacional (máquinas virtuais) disponibilizada pela RNP e a plataforma FIWARE-Lab@RNP. O caso de uso é lixeiras inteligentes para coleta de dados acerca da quantidade de resíduos orgânicos e monitoramento de níveis de lixo das lixeiras seletivas.

\section{Introdução}

A implantação de serviços avançados de Internet das Coisas (Internet of Things - IoT) exige requisitos rigorosos de comunicação, que serão alcançados com a 5G. A importância de IoT é praticamente inquestionável, em parte, devido à enorme quantidade de aplicações disponíveis e em desenvolvimento. Apesar da implantação e uso de um grande número de dispositivos de IoT já ser uma realidade, o tema ainda é considerado extremamente importante tanto para a indústria quanto para a academia.

Dentre as aplicações para IoT, aparece como destaque os ambientes inteligentes, e.g., cidade e Campus Inteligente. O Campus pode ser visto como uma versão reduzida 
da cidade, com várias características semelhantes a ela, por exemplo, é um ambiente com intensa mobilidade de pessoas e há questões relacionadas à mobilidade, estacionamento, segurança, alimentação, convívio social, coleta de lixo inteligente, etc.

O objetivo deste trabalho é apresentar e descrever os detalhes da implantação e os experimentos de integração do SOFTWAY4IoT realizado junto à infraestrutura computacional (máquinas virtuais - VMs) disponibilizada pela Rede Nacional de Ensino e Pesquisa (RNP) e a plataforma FIWARE-Lab@RNP. A implementação do caso de uso das lixeiras inteligentes [Oliveira-Jr et al. 2019] envolveu a Universidade Federal de Goiás (UFG), Universidade Federal do Rio Grande do Norte (UFRN), Universidade de Brasília (UNB) e Instituto Nacional de Telecomunicações (Inatel) no contexto do projeto 5G-IoT financiado pelo Ministério da Ciência, Tecnologia e Inovação do Brasil (MCTI) e RNP.

Aplicações no contexto de integração FIWARE-Lab@RNP e SOFTWAY4IoT foram desenvolvidas para testes de integração. Inicialmente foi desenvolvida uma aplicação que realiza o acionamento de um motor de forma remota e uma aplicação que faz envio das medições de um sensor ultrassônico. Para o processo de integração utilizamos duas APIs que são responsáveis por registrar os dispositivos na plataforma FIWARELab@RNP e intermediar a comunicação com os dispositivos. Esta construção de arquitetura possibilita a utilização dos elementos integradores de maneira desacoplada, onde o único componente FIWARE Lab presente na infraestrutura do SOFTWAY4IoT é o IoT Agent. Essa decisão de projeto torna a solução mais flexível onde os elementos Orion Context Broker e MongoDB podem ser implantados de forma independente.

Para além dessa Introdução, a Seção 2 apresenta brevemente o SOFTWAY4IoT e FIWARE-Lab@RNP, enquanto que a Seção 3 descreve a proposta de integração do SOFTWAY4IoT com a plataforma FIWARE-Lab@RNP. A Seção 4 apresenta os experimentos e testes realizados no contexto da integração envolvendo um caso de uso de lixeiras inteligentes no contexto de campus inteligente. Por fim, a Seção 5 apresenta as conclusões destacando as lições aprendidas, pontos de melhorias e sugestões de evolução.

\section{SOFTWAY4IoT e FIWARE-Lab@RNP}

O SOFTware-defined gateWAY and fog computing for IoT (SOFTWAY4IoT) [SOFTWAY4IoT 2021], [Cardoso et al. 2019], [Oliveira-Jr et al. 2020] é uma solução de comunicação IoT totalmente em software, aberta, virtualizada, integrada com computação em nuvem e com suporte a conectividade 5G. O SOFTWAY4IoT, por meio de seus 4 módulos principais, está registrado como programa de computador no Instituto Nacional da Propriedade Industrial (INPI) [Cardoso et al. a], [Cardoso et al. b], [Cardoso et al. c], [Cardoso et al. d] e disponível na prateleira de serviços IoT experimentais da RNP.

O SOFTWAY4IoT aborda quatro problemas principais: (i) necessidade de suportar múltiplas tecnologias de comunicação, e.g., WiFi, ZigBee, LoRa, nRF24; (ii) exposição de dispositivos de IoT à Internet pública, i.e., riscos à segurança dos dispositivos; (iii) conectividade dos dispositivos de IoT com a infraestrutura de computação em nuvem; (iv) implantação e gerência de múltiplos gateways dado um ambiente corporativo com múltiplos inquilinos e uma equipe enxuta de operação. O SOFTWAY4IoT foi desenvolvido tendo como base uma arquitetura que pode ser decomposta em dois elementos distintos, Gateway Manager e Gateways. Cada um desses dois elementos possui artefatos de software que desempenham papéis específicos. 
Além disso, a implementação baseia-se em Rádio Definido por Software (SDR), possibilitando múltiplas tecnologias de comunicação sem fio com apenas uma interface e um módulo híbrido para implementação de diferentes interfaces físicas. Por considerar o conceito de Redes Definidas por Software (SDN), há a possibilidade de fatiamento virtual de recursos de rede (network slicing) e é compatível com ecossistemas SDN sofisticados, e.g., OpenDayLigh. O suporte à segurança de redes dos dispositivos IoT inclui funcionalidades como NAT/Firewall e IDS, além de um módulo denominado PhySec para segurança na camada física utilizando o SDR, que possibilita a identificação radiométrica de dispositivos IoT. No que tange aos recursos de computação na borda, a tecnologia de Fog Computing é utilizada, permitindo a execução das aplicações virtualizadas sobre fatias de recursos (slices) da infraestrutura de Fog e/ou de Cloud Computing.

A plataforma FIWARE é uma plataforma genérica e aberta, é desenvolvida pela Comunidade Europeia e tem como principal objetivo contribuir para o desenvolvimento de aplicações para Internet do Futuro. O FIWARE é implantado em uma rede distribuída geograficamente de nós federados do FIWARE Lab. Cada nó do FIWARE Lab é mapeado para uma rede de data centers em cima do qual uma instância do OpenStack foi implantada, federada e configurada como um nó do FIWARE Lab (região da nuvem) operado por uma organização específica. Os nós do FIWARE Lab não fornecem apenas recursos de nuvem. Eles fazem parte dos ecossistemas FIWARE locais e dos iHubs FIWARE, apoiando e promovendo o acesso à tecnologia FIWARE.

O FIWARELab@RNP [Dantas et al. 2019, Dantas 2020] é um laboratório virtual e remoto para prototipação e experimentação de aplicações de IoT baseadas na plataforma FIWARE. Assim, o FIWARELab@RNP tem como principal objetivo permitir de modo transparente e completo o uso de recursos da FIWARE de forma descentralizada, permitindo o uso dos recursos sem a necessidade de se operar uma instância FIWARE local no ambiente de desenvolvimento do usuário. Entre suas funcionalidades estão a fácil criação, configuração e gerenciamento de instâncias de componentes FIWARE, implantação, controle e gerenciamento de dispositivos e serviços.

\section{Integração SOFTWAY4IoT e FIWARE-Lab@RNP}

Esta seção descreve os detalhes de integração entre os elementos da plataforma FIWARELab@RNP e o SOFTWAY4IoT. O processo de integração é executado através do desenvolvimento de duas APIs. A primeira é denominada SW4IoT-FIWARE-Manager, sendo responsável por operações de registro de dispositivos no IoT Agent da plataforma FIWARE. A segunda é denominada devicesApp e será responsável por intermediar a comunicação entre dispositivos IoT e o IoT Agent da FIWARE. Para implementar o processo de integração, serão utilizados os seguintes componentes FIWARE:

- ORION - responsável por gerenciar dados de contexto gerados por dispositivos IoT, oferecendo operações de gerenciamento e consulta desses mesmos dados via API RESTful.

- IoT Agent - funciona como um gateway de tradução de protocolo, convertendo os dados de diferentes tipos de protocolo para o formato de dados NGSI, o qual é utilizado pelo ORION em todas suas interações. Nessa integração ele fará uma ponte entre o formato de dados JSON e o NGSI. 
Esses dois componentes FIWARE utilizam uma instância do gerenciador de banco de dados MongoDB para persistência de dados. ORION utiliza MongoDB para armazenar dados de contexto gerados por aplicações e/ou dispositivos, e o IoT Agent utiliza esse gerenciador para armazenar informações sobre dispositivos IoT.

\subsection{Arquitetura}

O processo de integração é realizado através de um conjunto de interações entre componentes específicos da FIWARE-Lab@RNP e do SOFTWAY4IoT. A Figura 1 apresenta a arquitetura de integração, exibindo as interações entre os componentes ORION, IoT Agent e o MongoDB, todos da FIWARE, e os componentes WebSM, SW4IoTFIWARE-Manager, devicesApp FIWARE e os diferentes drivers de protocolo utilizados para comunicação com dispositivos IoT (do SOFTWAY4IoT).

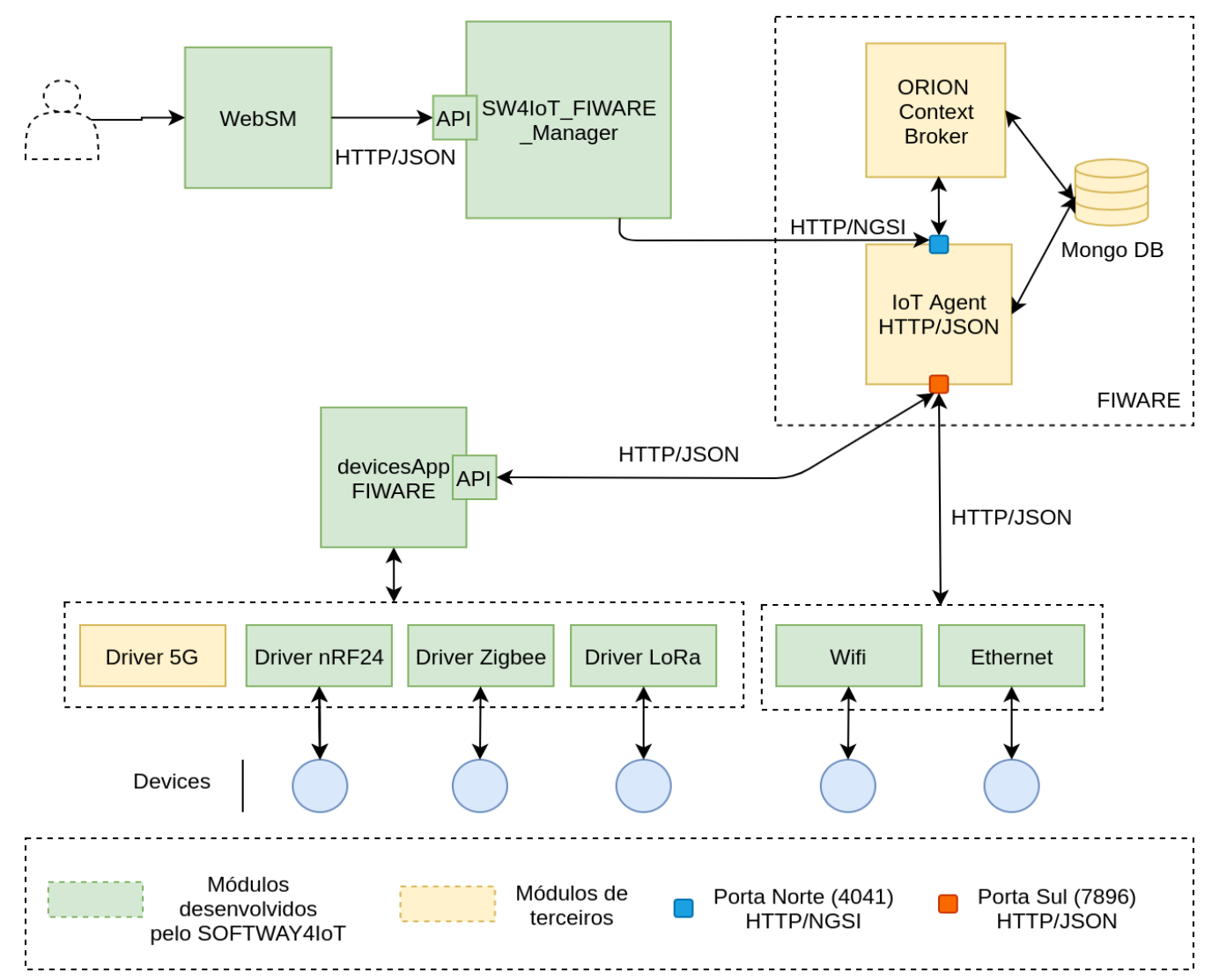

Figura 1. Arquitetura da integração do SOFTWAY4loT com a plataforma FIWARELab@RNP.

As seções a seguir apresentam em detalhes as características de cada um desses componentes.

\subsection{Componentes SOFTWAY4IoT}

O SW4IoT-FIWARE-Manager é o componente responsável pelo gerenciamento de dispositivos junto ao IoT Agent, o qual provê uma API RESTful para esse gerenciamento. Através desse componente é possível realizar as seguintes operações: registrar um novo dispositivo, alterar, excluir e obter informações de dispositivos já registrados. Ele atua 
encaminhando os dados informados via interface Web do WebSM para o registro no IoT Agent.

WebSM é a ferramenta de administração do SOFTWAY4IoT para múltiplos gateways IoT, a qual possibilita criar slices (fatias de recursos), associá-los aos gateways, além de adicionar, editar e remover dispositivos de comunicação sem fio e aplicações associadas às slices.

Os drivers são responsáveis por lidar com as especificidades dos protocolos de comunicação sem fio que não implementam toda a pilha TCP/IP e, por essa razão, não conseguem estabelecer comunicação diretamente com a internet. Para que o processo de comunicação aconteça, faz-se necessário a existência de um elemento intermediário (driver). Esse elemento é o responsável por realizar o processo de tradução entre o protocolo de comunicação original do dispositivo e o TCP/IP (padrão de comunicação para a Internet).

O 5G é o padrão de tecnologia de quinta geração para redes móveis. O Driver $5 \mathrm{G}$ é responsável por prover conexão e comunicação com dispositivos IoT através de redes 5G. O nRF24 é um módulo transceptor de baixo custo que opera na faixa ISM 2.4 GHz. O Driver nRF24 é responsável por lidar com as especificidades do protocolo de comunicação sem fio utilizado pelo módulo nRF24. O Zigbee é um protocolo (IEEE 802.15.4) desenvolvido para criação de redes de baixo consumo e custo. O Driver Zigbee é responsável por lidar com as especificidades do protocolo de comunicação sem fio Zigbee. LoRa é uma tecnologia de comunicação sem fio que permite comunicação a longas distâncias, com um consumo mínimo de energia. O Driver LoRa é responsável por lidar com as especificidades do protocolo de comunicação sem fio LoRa.

Na plataforma FIWARE, existe um IoT Agent específico para lidar com as particularidades de cada protocolo. Como os drivers do SOFTWAY4IoT já desempenham essa função, optamos por utilizá-los, eliminando a necessidade de incluir na arquitetura um IoT Agent para cada tipo de protocolo. Assim, a devicesApp foi desenvolvida para intermediar a comunicação entre os dispositivos via driver e o IoT Agent da FIWARE, atuando no repasse de comandos provenientes do IoT Agent destinados aos dispositivos IoT atuadores e no repasse de medições geradas por dispositivos IoT sensores destinadas ao IoT Agent.

\subsection{Componentes FIWARE}

ORION Context Broker é responsável pelo gerenciamento de dados de contexto das aplicações que utilizam a plataforma FIWARE, disponibilizando serviços de consulta e atualização desses dados através de uma API NGSI. Existem apenas dois tipos possíveis de interações de dados entre IoT Agent e ORION: as interações queryContext e updateContext. A queryContext é utilizada para operações de consulta e a updateContext para operações de atualização. Nessa integração, o ORION é utilizado para o gerenciamento de dados gerados por dispositivos IoT devidamente registrados no SOFTWAY4IoT, sejam eles sensores ou atuadores. Não foi necessário realizar mudanças no ORION para a integração.

IoT Agent é responsável por intermediar a comunicação entre dispositivos IoT e ORION, traduzindo os dados de diferentes tipos de protocolo para para o formato NGSI, 
o qual é utilizado pelo ORION em todas suas interações. Nessa integração, será utilizado o IoT Agent for JSON, o qual atua como uma ponte entre o protocolo JSON e o NGSI.

O tráfego que flui através do IoT Agent é dividido em dois tipos: tráfego Southbound e Northbound. Todo tráfego gerado pelo ORION em direção a um dispositivo atuador é chamado de tráfego Southbound, que consiste no envio de requisições de comando geradas pelo ORION em direção a um dispositivo IoT. O tráfego Northbound consiste no envio de medições geradas por sensores em direção ao ORION.

Para que um dispositivo possa receber comandos ou enviar medições, é necessário registrá-lo no IoT Agent. A esse processo dá-se o nome de provisionamento. Ao registar um dispositivo, seja um sensor ou atuador, devemos informar: o seu ID; sua entidade de dados, que é a forma como um dispositivo é representado dentro da plataforma FIWARE; o tipo da entidade; os atributos que correspondem ao valor de leitura de cada sensor e os comandos disponíveis para cada atuador, sendo também necessário informar o endpoint (URL) no qual o dispositivo está atendendo à requisições.

Na integração entre FIWARE-Lab@RNP e SOFTWAY4IoT, a comunicação com os dispositivos IoT é realizada de acordo com a tecnologia de comunicação utilizada pelos mesmos. Para os dispositivos que utilizam tecnologias de comunicação que implementam toda a pilha TCP/IP, e.g., Wi-Fi e Ethernet, o processo de comunicação é realizado diretamente entre o respectivo dispositivo e o IoT Agent. Para esses casos, não é necessário a utilização do nenhum mecanismo para interfacear a comunicação, pois os respectivos dispositivos possuem a capacidade de atender as requisições HTTP de forma direta. Para os dispositivos que utilizam tecnologias de comunicação que não implementam toda a pilha TCP/IP, e.g., Lora, Zigbee, nRF24, o processo de comunicação é realizado através da utilização de um agente intermediário, responsável por interfacear a comunicação entre o respectivo dispositivo e o IoT Agent. Nesse cenário, a devicesApp e os drivers, são os responsáveis por intermediar o processo de comunicação, realizando a tradução entre o protocolo original do dispositivo e o HTTP.

Dessa forma, a comunicação com os dispositivos IoT é um dos pontos principais das vantagens da integração entre FIWARE-Lab@RNP e SOFTWAY4IoT, pois nos proporciona melhorias na criação, configuração e gerenciamento de instâncias de componentes FIWARE, permite uma melhor implantação, replicação e controle dos componentes do SOFTWAY4IoT, e melhor gerenciamento dos dispositivos e serviços instanciados no SOFTWAY4IoT.

\section{Experimentos e Testes da Integração}

O processo de integração foi implementado através do desenvolvimento de duas APIs, responsáveis por registrar os dispositivos no IoT Agent da plataforma FIWARE-Lab@RNP e intermediar a comunicação entre dispositivos IoT (coisas - things) e o IoT Agent. A arquitetura utilizada na integração entre o SOFTWAY4IoT e a plataforma FIWARE Lab, possibilita a utilização dos elementos integradores de maneira desacoplada. O único componente FIWARE Lab presente na infraestrutura do SOFTWAY4IoT é o IoT agent. Assim, os elementos Orion Context Broker e MongoDB podem ser implantados de forma independente.

Na etapa de implantação para realização de testes é importante observar o comportamento dos componentes do SOFTWAY4IoT quando expostos em um ambiente de 
estresse, sob condições similares às que possivelmente serão encontradas quando do uso em produção. Para a realização do teste, a RNP disponibilizou em sua infraestrutura, máquinas virtuais (VMs) para as equipes envolvidas. Todas as máquinas virtuais foram disponibilizadas contendo elementos de software que obedecem os requisitos mínimos de execução do SOFTWAY4IoT. Os requisitos em questão estão descritos no Manual de Implantação [SOFTWAY4IoT 2021]. Além da disponibilização das máquinas virtuais, a RNP forneceu também equipamentos do tipo Mini PC para serem utilizados como gateways no SOFTWAY4IoT.

O ambiente físico, onde será implantada a solução para respectiva prova de conceito, foi definido como sendo a Universidade de Brasília (UNB) o ambiente a ser utilizado. Os artefatos de software produzidos pela UFG no âmbito do SOFTWAY4IoT, integrados com os artefatos produzidos pela UFRN no âmbito do FIWARE Lab, fazendo uso do rádio $5 \mathrm{G}$ do Inatel, devem prover infraestrutura de conexão, aplicado a um cenário de internet das coisas, voltado a um ambiente cujo posicionamento geográfico não possibilite cobertura por sinais que permitam conectividade com a internet.

\subsection{Implantação SOFTWAY4IoT}

O processo de implantação dos elementos de software do SOFTWAY4IoT tem como base o Ansible. Dos diversos artefatos de software que compõem a solução, alguns elementos integram um componente de orquestração (Gateway Manager), e os demais integram um componente de monitoramento e controle (Gateway). A implantação dos elementos SOFTWAY4IoT é realizada através da execução de um playbook de implantação Ansible. Esse playbook automatiza a execução de diversas ações a nível de infraestrutura, permitindo ainda escalar o processo de implantação para diversos equipamentos de forma concomitante.

Visando testar de forma efetiva o comportamento da solução, o playbook de implantação do Gateway Manager foi executado em ambas as máquinas virtuais disponibilizadas pela RNP. A execução de tal processo originou diversos ajustes e melhorias na referida solução. De forma similar, realizamos a implantação do elemento Gateway utilizando o equipamento Mini PC, e interligado o mesmo com o respectivo Gateway Manager implantado nas máquinas virtuais.

No processo de implantação do Gateway Manager testamos a integração com os componentes do FIWARE Lab. Nesse contexto, a integração descrita na Seção 3 permitiu a construção de uma estrutura de implantação parametrizada que possibilita adicionar componentes do FIWARE Lab em conjunto com os elementos do SOFTWAY4IoT. Em outras palavras, é possível implantar os elemento ORION e IOT Agent de forma automatizada juntamente com a Gateway Manager.

\subsection{Aplicações de Teste}

Após a implantação de todos os elementos de software que compõem as soluções envolvidas na infraestrutura computacional disponibilizada pela RNP, o próximo item a ser aferido está associado à concepção de aplicações que façam uso dessa infraestrutura e que estejam voltadas para o cenário de IoT. Nesse sentido foram desenvolvidas duas aplicações, conforme descrito a seguir. 


\subsubsection{Lixeiras Monitoradas}

A primeira aplicação desenvolvida tem como foco o monitoramento de lixeiras. Basicamente a aplicação em questão tem a responsabilidade de monitorar constantemente o volume de lixo contido em um determinado recipiente. O monitoramento em questão é realizado através da utilização de sensores ultrassônicos, conforme apresentado na Figura 2.

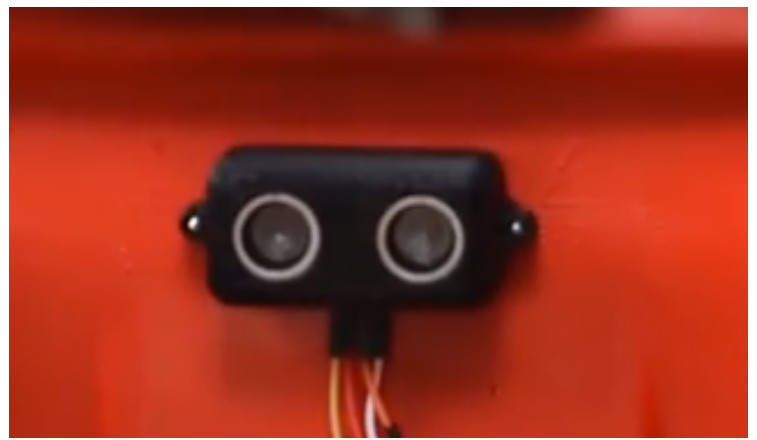

Figura 2. Sensor ultrassônico utilizado para o monitoramento do volume de lixo contido em um determinado recipiente de coleta.

Os sensores em questão, estão vinculados a um Gateway que foi devidamente implantado conforme descrito na Seção 4.1, e gerenciado por um Gateway Manager implantado na infraestrutura computacional da RNP. Os dados coletados pelos sensores são transmitidos pelo Gateway para um base de dados remota, e posteriormente apresentados em uma aplicação Web de monitoramento e controle conforme apresentado na Figura 3.

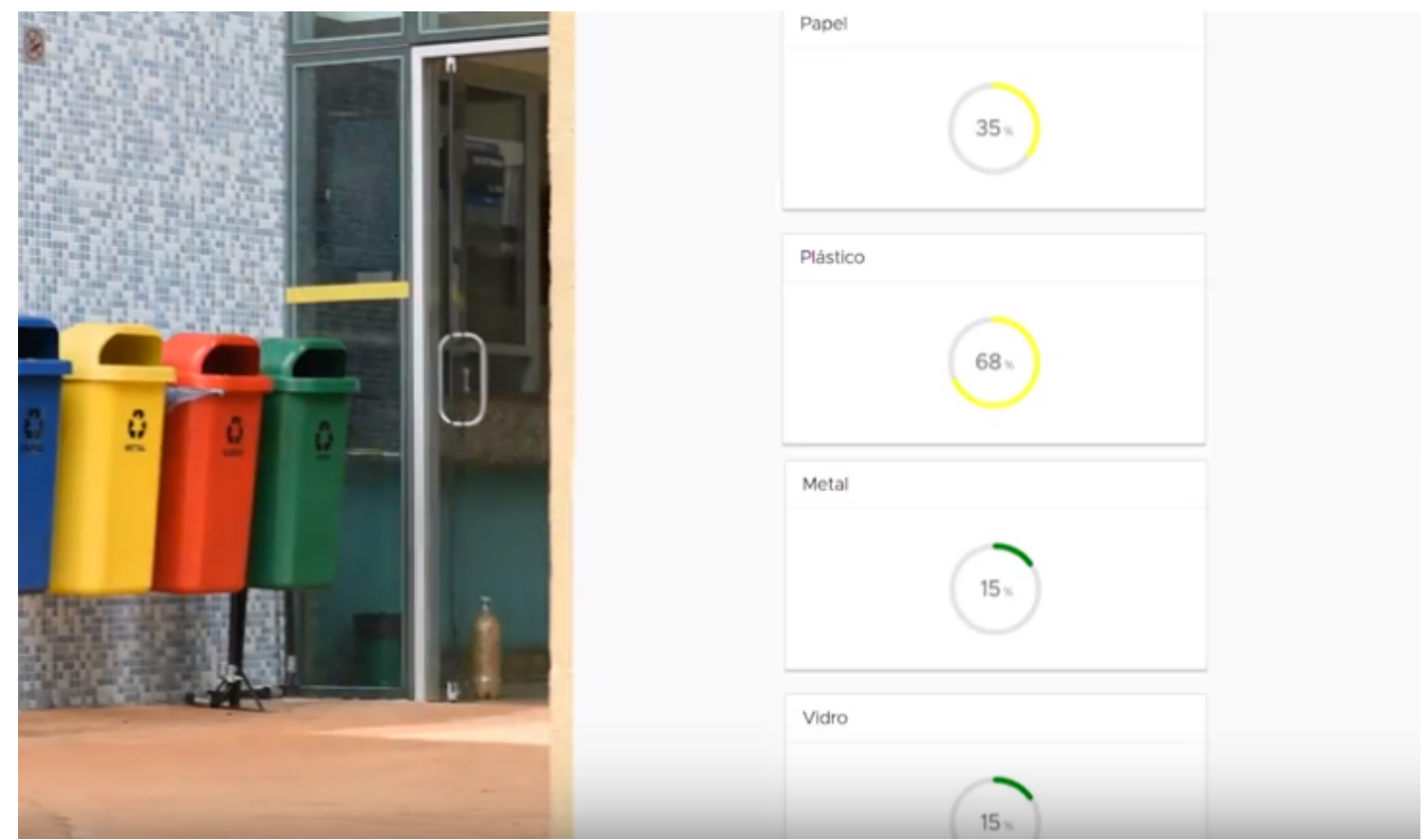

Figura 3. Aplicação Web para apresentação das informações coletadas pelos sensores ultrassônicos de monitoramento de lixeira. 
A equipe da UFG produziu um vídeo demonstrativo das lixeiras inteligentes apresentando todo o contexto da aplicação em questão (https://www.youtube.com/watch?v=m-YY09riDlk).

\subsubsection{Acionamento remoto de motor}

A aplicação apresentada na Seção 4.2.1 pode ser descrita como uma aplicação de fluxo unidirecional, em outras palavras, as informações partem dos sensores em direção à base de dados com posterior apresentação por parte de uma página Web.

Neste sentido, não é possível nenhuma interação no sentido oposto ao fluxo das informações. Partindo do princípio de que em um cenário de dispositivos IoT, eventuais aplicações demandam de um fluxo bidirecional, como por exemplo, o acionamento remoto, desenvolvemos uma aplicação que realiza a coleta de dados através de um sensor ultrassônico e ao mesmo tempo possibilita acionar remotamente um motor. Os respectivos sensores, bem como o motor ficam conectados a um gateway controlado por um Gateway Manager.

Todas as informações acerca dos parâmetros que possibilitam o acionamento remoto do respectivo dispositivo são controlados pelo FIWARE Lab com interfaceamento realizado através do SOFTWAY4IoT. A construção da aplicação em questão, envolve todos os elementos que compõem as soluções SOFTWAY4IoT e FIWARE-Lab@RNP. O objetivo principal é testar a integração realizada entre os artefatos de software que ocorreu conforme o esperado.

Maiores detalhes acerca do processo de integração pode ser obtido através do documento intitulado Manual de uso - Integração FIWARE-Lab@RNP que pode ser disponibilizado pela RNP. O acesso ao código fonte da respectiva aplicação, bem como à documentação está devidamente acondicionada no GitLab RNP no repositório App de Acionamento Remoto.

\section{Conclusões}

Este trabalho apresenta os detalhes da implantação e os experimentos de integração de um gateway IoT definido por software (SOFTWAY4IoT) realizado junto à infraestrutura computacional disponibilizada pela RNP e a plataforma FIWARE-Lab@RNP. Os resultados apresentados demonstram a capacidade da solução em gerenciar dispositivos IoT de diferentes tecnologias e em diferentes localizações geográficas e em escala.

Para os experimentos foram executadas aplicações de testes de integração, sendo elas as lixeiras monitoradas e o acionamento de um motor de forma remota. Para o processo de integração utilizamos duas APIs que são responsáveis por registrar os dispositivos na plataforma FIWARE-Lab@RNP e intermediar a comunicação com os dispositivos. A arquitetura construída possibilita a utilização dos elementos integradores de maneira desacoplada, onde o único componente FIWARE Lab presente na infraestrutura do SOFTWAY4IoT é o IoT Agent, o que sem dúvidas é um ponto positivo da solução. Pois, tira do ambiente do usuário a necessidade de uma instância local do FIWARE Lab completa e melhora a usabilidade dos serviços do FIWARE.

A comunicação com o FIWARE-Lab@RNP referencia as respectivas portas das 
instâncias em execução no FIWARE-Lab@RNP (Orion e MongoDB) no arquivo de configuração do IoT Agent em execução na infra do SOFTWAY4IoT. Com relação ao middleware, o IoT agent serve como um túnel, cada protocolo é tratado de forma individual pela devicesApp e o respectivo driver do protocolo. Adicionalmente, vantagens do uso do tesbed seria os benefícios de se ter o infra do FIWARE-Lab@RNP em nuvem. A possibilidade de se criar, implantar múltiplas instâncias de cada componente da FIWARE-Lab@RNP de forma facilitada.

Outro ponto importante observado nos experimentos foi a replicação da integração realizada por equipes diferentes no decorrer do projeto. Todos os componentes da integração entre FIWARE-Lab@RNP e SOFTWAY4IoT foram replicados por times de desenvolvimentos diferentes a fim de auxiliar no desenvolvimento de aplicações em outro campus inteligente. Equipes de desenvolvimento da UFG juntamente com a equipe da UNB conseguiram replicar com êxito a implantação dos componentes de integração FIWARE-Lab@RNP e SOFTWAY4IoT e sucesso no teste com novas aplicações no Campus em Brasília. Assim, é possível que qualquer equipe consiga reproduzir os experimentos e adaptá-los conforme os objetivos específicos.

Como pontos de melhoria, podem ser necessários para uma ampla utilização do FIWARE-Lab@RNP, uma vez que, até o momento, é um nó isolado e não faz parte da rede distribuída geograficamente do FIWARE-Lab Europeu. Entre eles estão melhorias no processo de configuração, para que aplicações complexas sejam integradas e utilizem a ferramenta como um todo. Melhorias no cadastramento para utilização da ferramenta, suportar a integração com ferramentas de acesso federado, como por exemplo, a Comunidade Acadêmica Federada (CAFe) também é um ponto interessante de melhoria e suporte a ferramentas de Cloud Computing, tais como AWS e Google Cloud Platform.

\section{Agradecimentos}

Este trabalho foi financiado pela RNP e MCTI no contexto do projeto 5G-IoT. Agradecemos UFG, UFRN, UNB e Inatel pela colaboração no desenvolvimento das soluções e experimentos.

\section{Referências}

Cardoso, K., Oliveira-Jr, A., and Correa, S. (2019). SOFTWAY4IoT: SOFTware-defined gateWAY and Fog Computing for IoT (Internet of Things). White paper, Workshop RNP-Programa de Internet Avançada.

Cardoso, K. V., Oliveira-Jr, A., Correa, S. L., Macedo, C. J. A., Almeida, G. M. F., and Sousa, P. F. A. Gereiot - gerenciador de redes virtuais para sistema corporativo de gateways iot. In INPI - Instituto Nacional da Propriedade Industrial, number BR n. 512020002004-6. 25 sep. 2020.

Cardoso, K. V., Oliveira-Jr, A., Correa, S. L., Macedo, C. J. A., Almeida, G. M. F., and Sousa, P. F. A. Givsiot - gerenciador de interfaces virtuais sem fio para comunicação iot. In INPI - Instituto Nacional da Propriedade Industrial, number BR n. 512020002005-4. 25 sep. 2020.

Cardoso, K. V., Oliveira-Jr, A., Correa, S. L., Macedo, C. J. A., Almeida, G. M. F., and Sousa, P. F. A. Icsgwiot - interface de comunicação entre sistema corporativo de 
gateways iot e plataforma de desenvolvimento de aplicações iot. In INPI - Instituto Nacional da Propriedade Industrial, number BR n. 12020002006-2. 25 sep. 2020.

Cardoso, K. V., Oliveira-Jr, A., Correa, S. L., Macedo, C. J. A., Almeida, G. M. F., and Sousa, P. F. A. Orqcgiot - orquestrador de componentes virtuais para sistema corporativo de gateways iot. In INPI - Instituto Nacional da Propriedade Industrial, number BR n. 512020002007-0. 25 sep. 2020.

Dantas, L., Cavalcante, E., and Batista, T. (2019). A development environment for fiwarebased internet of things applications. Proceedings of the 6th International Workshop on Middleware and Applications for the Internet of Things, pages 21-26.

Dantas, L. C. C. (2020). A Virtual Laboratory for Developing and Experimenting Internet of Things Applications. Master's thesis, Universidade Federal do Rio Grande do Norte (UFRN).

Oliveira-Jr, A., Cardoso, K., Sousa, F., and Moreira, W. (2020). A lightweight slice-based quality of service manager for iot. MDPI IoT, 1:49-75.

Oliveira-Jr, A. C., Cardoso, K. V., Corrêa, S. L., and Moreira, W. (2019). Internet das coisas para aplicações sustentáveis. Computação Brasil, (40):14-18.

SOFTWAY4IoT (2021). Software defined gateway and fog computing for internet of things (softway4iot), https://softway4iot.labora.inf.ufg.br/ (acessado em março 2021). 